\title{
Diffractive stacks of metamaterial lattices with a complex unit cell: Self-consistent long-range bianisotropic interactions in experiment and theory
}

\author{
Andrej Kwadrin* and A. Femius Koenderink \\ Center for Nanophotonics, FOM Institute AMOLF, Science Park 104, 1098 XG Amsterdam, The Netherlands
}

(Received 20 November 2013; revised manuscript received 13 December 2013; published 15 January 2014)

\begin{abstract}
Metasurfaces and metamaterials promise arbitrary rerouting of light using two-dimensional (2D) planar arrangements of electric and magnetic scatterers, respectively, 3D stacks built out of such 2D planes. An important problem is how to self-consistently model the response of these systems in a manner that retains dipole intuition yet does full justice to the self-consistent multiple scattering via near-field and far-field retarded interactions. We set up such a general model for metamaterial lattices of complex 2D unit cells of poly-atomic basis as well as allowing for stacking in a third dimension. In particular, each scatterer is quantified by a magnetoelectric polarizability tensor and Ewald lattice summation deals with all near-field and long-range retarded electric, magnetic, and magnetoelectric couplings self-consistently. We show in theory and experiment that grating diffraction orders of dilute split ring lattices with complex unit cells show a background-free signature of magnetic dipole response. For denser lattices experiment and theory show that complex unit cells can reduce the apparent effect of bianisotropy, i.e., the strong oblique-incidence handed response that was reported for simple split ring lattices. Finally, the method is applied to calculate transmission of finite stacks of lattices. Thereby our simple methodology allows us to trace the emergence of effective material constants when building a 3D metamaterial layer by layer, as well as facilitating the design of metasurfaces.
\end{abstract}

DOI: 10.1103/PhysRevB.89.045120

PACS number(s): 78.67.Pt, 81.05.Xj, 42.30.Kq

\section{INTRODUCTION}

In the field of metamaterials, there is a growing interest in metasurfaces [1,2]: two-dimensional (2D) arrangements of subwavelength scatterers which combine a strong electric and magnetic response to electromagnetic fields, both of which may contribute equally to scattering $[1,3,4]$. These lattice arrangements offer an unprecedented level of control over the reflection, transmission, and refraction of light as seen in state-of-the-art phase mask [5] and phased array antenna designs [6,7]. While metasurfaces are typically 2D sheets of nonidentical scatterers forming a complex repeated unit cell, metamaterials, in turn, can be conceptually viewed as a 3D stack of 2D lattices of identical meta-atoms [8,9]. Exactly how the collective response of a metasurface or metamaterial comes about is a function, first, of the scattering properties of individual building blocks and, second, how a multitude of possibly nonidentical building blocks are arranged to cover a surface [10,11]. Exactly how the magnetic and electric scattering of single building blocks comes about has been a topic of intense discussion, centering mainly on electric dipole models for plasmonics and LC-circuit models [9,12-15] for metamaterial atoms such as split rings. LC-circuit intuition directly implies electric and magnetic dipole-dipole interaction, as well as "bianisotropic" magnetoelectric cross-coupling terms [14-16]. Given this single-building-block understanding, an important question currently faced by designers of metasurfaces and metamaterials is how to deal with complex 2D lattices and lattice stacks, while accounting for both electric and magnetic interactions between such building blocks.

In this article, we set up an analytical theory based on Ewald lattice summation [17] that can predict the response of diffractive as well as nondiffractive 2D periodic lattices with

*kwadrin@amolf.nl complex unit cells, as well as stacks of such gratings that form a $3 \mathrm{D}$ structure, taking as input the magnetoelectric polarization tensors of the magnetoelectric scatterers that form the unit cell. In order to test this model, we experimentally verify the optical response of 2D lattices of split rings, since for split rings the single-object polarizability is well known [14,18-20]. In particular, we examine both dilute and dense lattices of two symmetries, i.e., square lattices in which all single split rings are arranged with their slits pointing in the same direction (A lattice; Fig. 1, left), respectively, in which slit orientations (B lattice) alternate. This choice is motivated by experimental studies on low-symmetry planar arrays initiated by Decker et al. [21]. The dilute lattices are designed to show grating diffraction orders which we examine for magnetic signatures. In particular, we predict and observe additional diffraction orders for the B lattice compared to the A lattice that directly reflect purely magnetic dipole contributions. As the second test we examine the transmission of dense, i.e., nondiffractive lattices, focusing, in particular, on oblique-incidence circularly polarized input and detection. This geometry provides a measure of bianisotropy or "pseudochirality." We observe cancellation of the bianisotropy present in each building block for the collective response of the B lattice. Finally, we demonstrate that our calculation methodology has great potential for resolving the fundamental question of how effective medium parameters emerge from the stacking of building blocks into 3D slabs, by demonstrating that one can self-consistently calculate reflection and transmission amplitudes that can be used as input for retrieval algorithms. The unique property of our approach is that it is very simple, and allows arbitrary magnetoelectric response per building block, yet accounts for all the complex self-consistent retarded interactions, both in the plane of periodicity and transverse to the stacked planes, that define the debate on how to deal with spatial dispersion and bianisotropy in the resulting effective medium. 

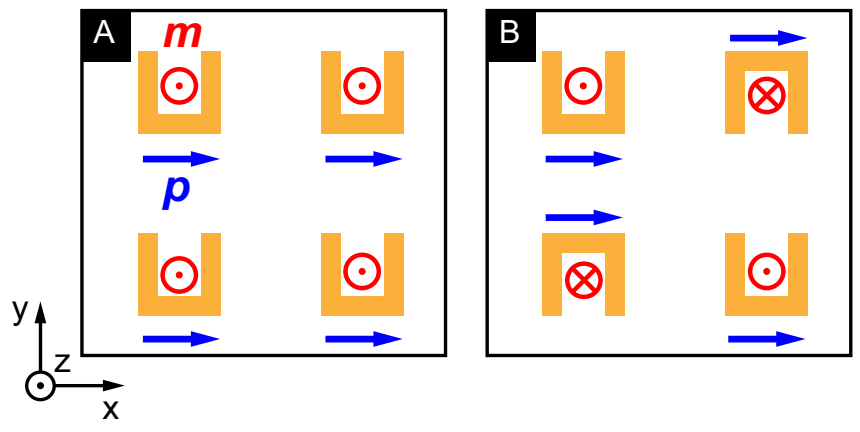

FIG. 1. (Color online) Nonprimitive unit cells with equal (A) and alternating (B) split ring orientation. An x-polarized electric driving field sets an electric dipole moment $\mathbf{p}$ for each individual split ring. Depending on the orientation of the respective split ring, a magnetic dipole moment $\mathbf{m}$ will be acquired. The electric dipole orientation will be the same for unit cells $\mathrm{A}$ and $\mathrm{B}$, namely, in the $+x$ direction. However, the magnetic dipoles, while being uniformly $+z$-oriented for unit cell $\mathrm{A}$, will be alternating in the $+z$ and $-z$ orientations for unit cell B.

This article is structured as follows. Sections II and III describe the theory. Section IV reports grating diffraction calculations for the two lattice symmetries. Section V covers the Fourier microscopy setup that was utilized to gather diffraction patterns discussed in Sec. VI. Angle-resolved transmission experiments conducted on nondiffractive lattices are described and compared to analytical point dipole (lattice sum) calculations in Sec. VII. In Sec. VIII, we apply our point dipole model to calculate transmission and reflection for finite stacks of 2D metamaterial layers.

\section{STARTING POINT: 2D LATTICE SUM THEORY}

The main point of this paper is to take the point dipole model reported in Ref. [14] and show how to extend it to arbitrary stacks and supercells of 2D lattices. In a point scattering model [22], the induced electric and magnetic point dipole moments $\mathbf{p}$ and $\mathbf{m}$, in response to incident electric and magnetic fields $\mathbf{E}_{\mathrm{in}}$ and $\mathbf{H}_{\mathrm{in}}$, are set by the point scatterer's polarizability $\boldsymbol{\alpha}$ according to

$$
\left(\begin{array}{c}
\boldsymbol{p} \\
\boldsymbol{m}
\end{array}\right)=\alpha\left(\begin{array}{c}
\boldsymbol{E}_{\mathrm{in}} \\
\boldsymbol{H}_{\mathrm{in}}
\end{array}\right)
$$

For completeness, we briefly recapitulate how one can deal with an arrangement of such scatterers in a simple 2D lattice, defined by lattice vectors $\mathbf{R}_{m n}=m \mathbf{a}_{1}+n \mathbf{a}_{2}$ with integer $m$ and $n$ and real-space basis vectors $\mathbf{a}_{1,2}$. The response of a point scatterer at position $\mathbf{R}_{m n}$ is self-consistently set by the incident field, plus the field of all other dipoles in the lattice, according to

$$
\begin{aligned}
\left(\begin{array}{c}
\boldsymbol{p}_{m n} \\
\boldsymbol{m}_{m n}
\end{array}\right)= & \alpha\left[\left(\begin{array}{c}
\boldsymbol{E}_{\mathrm{in}}\left(\mathbf{R}_{m n}\right) \\
\boldsymbol{H}_{\mathrm{in}}\left(\mathbf{R}_{m n}\right)
\end{array}\right)\right. \\
& \left.+\sum_{m^{\prime} \neq m, n^{\prime} \neq n} \mathbf{G}^{0}\left(\mathbf{R}_{m n}-\mathbf{R}_{m^{\prime} n^{\prime}}\right)\left(\begin{array}{c}
\boldsymbol{p}_{m^{\prime} n^{\prime}} \\
\boldsymbol{m}_{m^{\prime} n^{\prime}}
\end{array}\right)\right] .
\end{aligned}
$$

If we take a plane wave incident with parallel wave vector $\mathbf{k}_{\|}$ (in the first Brillouin zone) and use the translation invariance of the lattice, we can substitute a Bloch wave form $\left(\boldsymbol{p}_{m n}, \boldsymbol{m}_{m n}\right)^{T}=$ $e^{i \mathbf{k}_{\| \mid} \cdot \mathbf{R}_{m n}}\left(\boldsymbol{p}_{00}, \boldsymbol{m}_{00}\right)^{T}$ to obtain

$$
\left(\begin{array}{c}
\boldsymbol{p}_{00} \\
\boldsymbol{m}_{00}
\end{array}\right)=\left[\boldsymbol{\alpha}^{-1}-\mathcal{G}^{\neq}\left(\mathbf{k}_{\|}, 0\right)\right]^{-1}\left(\begin{array}{c}
\boldsymbol{E}_{\mathrm{in}}\left(\mathbf{R}_{00}\right) \\
\boldsymbol{H}_{\mathrm{in}}\left(\mathbf{R}_{00}\right)
\end{array}\right) .
$$

Here, $\mathcal{G}^{\neq}\left(\mathbf{k}_{\|}, 0\right)$ is a summation of the free-space $6 \times 6$ dyadic Green function $\mathbf{G}^{0}$ over all positions on the $2 \mathrm{D}$ periodic realspace lattice barring the origin:

$$
\mathcal{G}^{\neq}\left(\mathbf{k}_{\|}, \mathbf{r}\right)=\sum_{m, n \neq 0} \mathbf{G}^{0}\left(\mathbf{R}_{m n}-\mathbf{r}\right) e^{i \mathbf{k}_{\| \mid} \cdot \mathbf{R}_{m n}} .
$$

We refer to the summation without exclusion of $m=n=0$ as $\mathcal{G}\left(\mathbf{k}_{\|}, \mathbf{r}\right)$. The necessary steps for $6 \times 6$ dyadic Green functions are easily derived from the known scalar Green function lattice sum. We refer to the Appendix and our recent report [23,24] for implementation details. As a subtle point we note that throughout this work $\mathbf{R}_{m n}$ represent reciprocal lattice vectors strictly in the $z=0$ plane, while the "observation point" $\mathbf{r}=(x, y, z)=\left(\mathbf{r}_{\|}, z\right)$ at which the lattice sum is evaluated is anywhere in three dimensions, i.e., at any $z$. Since each $\mathbf{G}^{0}\left(\mathbf{R}_{m n}-\mathbf{r}\right)$ is essentially a spherical wave, the lattice sum contains retardation both in the plane and perpendicular to the plane, even though only a phase factor depending on $\mathbf{k}_{\|}$is explicitly visible in Eq. (4).

Once one has obtained the induced dipole moments given the incident field, it is straightforward to calculate the nearfield distribution at any point inside or outside the lattice, i.e., at any $\mathbf{r}$ :

$$
\left(\begin{array}{c}
\mathbf{E}(\mathbf{r}) \\
\mathbf{H}(\mathbf{r})
\end{array}\right)=\left(\begin{array}{c}
\mathbf{E}_{\text {in }} \\
\mathbf{H}_{\text {in }}
\end{array}\right) e^{i \mathbf{k} \cdot \mathbf{r}}+\mathcal{G}\left(\mathbf{k}_{\|}, \mathbf{r}\right)\left(\begin{array}{c}
\mathbf{p}_{00} \\
\mathbf{m}_{00}
\end{array}\right) .
$$

Similarly, one can calculate the intensity and polarization of the diffracted, reflected, and transmitted waves. Using the completeness relation of the lattice, one retrieves diffracted orders in the far field of the form [17]

$$
\left(\begin{array}{l}
\mathbf{E}(\mathbf{r}) \\
\mathbf{H}(\mathbf{r})
\end{array}\right)=\sum_{\mathbf{g},\left|\mathbf{k}^{\mathbf{g}}\right| \leqslant k}\left(\begin{array}{l}
\mathbf{E}^{\mathbf{g}} \\
\mathbf{H}^{\mathbf{g}}
\end{array}\right) e^{i \mathbf{k}^{\mathrm{g}} \cdot \mathbf{r}},
$$

where the diffracted wave vectors are $\mathbf{k}^{\mathbf{g}}=$ $\left(\mathbf{k}_{\|}+\mathbf{g}, \pm \sqrt{k^{2}-\left|\mathbf{k}_{\|}+\mathbf{g}\right|^{2}}\right)$, which can be identified with polar diffraction angles $\theta, \phi$ through $\mathbf{k}^{\mathbf{g}}=$ $k(\cos \phi \sin \theta, \sin \phi \sin \theta, \cos \theta)$. Note that the diffracted orders are simply set by parallel wave vector conservation modulo the addition of any vector $\mathbf{g}$ from the reciprocal lattice. The fields associated with each order are

$$
\left(\begin{array}{l}
\mathbf{E}^{\mathbf{g}} \\
\mathbf{H}^{\mathbf{g}}
\end{array}\right)=\frac{2 \pi k i}{A \cos \theta} \mathbf{M}(\theta, \phi)\left(\begin{array}{c}
\mathbf{p}_{0} \\
\mathbf{m}_{0}
\end{array}\right) .
$$

Here $A$ is the unit cell area, and the orientation matrix $\mathbf{M}(\theta, \phi)$ can be written as

$$
\mathbf{M}=\left(\begin{array}{cc}
D & O \\
-O & D
\end{array}\right)
$$

where

$$
D=\left(\begin{array}{ccc}
1-x^{2} & -x y & -x z \\
-x y & 1-y^{2} & -y z \\
-x z & -y z & 1-z^{2}
\end{array}\right)
$$


and

$$
O=\left(\begin{array}{ccc}
0 & -z & y \\
z & 0 & -x \\
-y & x & 0
\end{array}\right),
$$

using as short hand $x=\cos \phi \sin \theta, y=\sin \phi \sin \theta$, and $z=$ $\cos \theta$. Since these are only scattered fields, one still needs to add the incident field to obtain the zero-order transmitted beam.

\section{COMPLEX BASE AND STACKS OF 2D LAYERS}

Having defined our starting point, we now turn to setting up a theory that can deal with almost arbitrary stacks of $2 \mathrm{D}$ lattices. To define the class of problems for which our approach is applicable more clearly, we outline how to deal with an arbitrarily large but finite set of $N$ lattices where each lattice has the same reciprocal lattice vectors but can have its base point arbitrarily shifted both in the plane of periodicity and transverse to it. Furthermore, each lattice can contain a different type of scatterer. Thereby, our theory as outlined in this section can directly deal with a rich variety of problems. These include the physics of planar structures with complicated supercells of many different scatterers, such as metasurfaces or stacks of metamaterial planes that form a 3D structure.

Suppose we have $m=1 \ldots N$ lattices of the same symmetry, each shifted by an arbitrary spatial offset $\mathbf{r}_{0,0}^{m}$ sideways, and $z^{m}$ perpendicular to the plane, and potentially each containing a different type of scatterer of polarizability $\boldsymbol{\alpha}_{m}$. As an example, lattice type B (Fig. 1, right) can be represented by two lattices (one for each split ring orientation) with a relative offset of $\sqrt{2} / 2$ in the $(x, y)$ plane. Since under our assumption Bloch's theorem still holds, the problem of $N$ infinite periodic lattices reduces to finding just the $N$ dipole moments on the central lattice sites in each lattice, i.e., the sites $\left(\mathbf{r}_{0}^{m}, z^{m}\right)$. Indeed, Bloch's theorem asserts that at any other lattice site in the same layer $m$, the moment is simply $\mathbf{p}^{m}\left(\mathbf{r}_{0}^{m}+\mathbf{R}, z^{m}\right)=$ $e^{i \mathbf{k}_{\|} \cdot \mathbf{R}} \mathbf{p}^{m}\left(\mathbf{r}_{0}^{m}, z^{m}\right)$. The $N$ independent dipole moments are once again set by a self-consistent equation. For the dipole moment at $\left(\mathbf{r}_{0}^{m}, z^{m}\right)$ in layer $m$, the self-consistent equation reads

$$
\begin{aligned}
\left(\begin{array}{c}
\boldsymbol{p}^{m} \\
\boldsymbol{m}^{m}
\end{array}\right)= & \boldsymbol{\alpha}_{m}\left[\left(\begin{array}{c}
\boldsymbol{E}_{\text {in }} \\
\boldsymbol{H}_{\text {in }}
\end{array}\right) e^{i \mathbf{k} \cdot\left(\mathbf{r}_{0}^{m}, z^{m}\right)}+\mathcal{G}^{\neq}\left(\mathbf{k}_{\|}, 0\right)\left(\begin{array}{c}
\boldsymbol{p}^{m} \\
\boldsymbol{m}^{m}
\end{array}\right)\right. \\
& \left.+\sum_{m^{\prime}=1, m^{\prime} \neq m}^{N} \mathcal{G}\left(\mathbf{k}_{\|},\left(\mathbf{r}_{0}^{m^{\prime}}-\mathbf{r}_{0}^{m}, z_{m^{\prime}}-z_{m}\right)\right)\left(\begin{array}{c}
\boldsymbol{p}^{m^{\prime}} \\
\boldsymbol{m}^{m^{\prime}}
\end{array}\right)\right] .
\end{aligned}
$$

Here, the interpretation is that any dipole is driven by the external incident field (first term on the right-hand side), by all the dipoles except itself in the same layer [second term on the right-hand side, which also occurs in Eq. (3)], and, moreover, by all the dipoles in all the other layers. In this third term a full lattice summation, including the $(0,0)$ term, occurs. It should be noted that in the first term, i.e., the direct driving, the retardation of the driving across the structure is directly explicit both transverse to the plane and in the plane, as the term $\mathbf{k} \cdot\left(\mathbf{r}_{0}^{m}, z\right)$ explicitly contains the full $3 \mathrm{D}$ wave vector and $3 \mathrm{D}$ lattice base point coordinate. What is less obvious upon inspection is that the third term, i.e., $\mathcal{G}\left(\mathbf{k}_{\|},\left(\mathbf{r}_{0}^{m^{\prime}}-\mathbf{r}_{0}^{m}, z_{m^{\prime}}-z_{m}\right)\right)$, also accounts for retardation both in plane (evident through the explicit argument $\mathbf{k}_{\|}$) and out of plane, i.e., for phase increments across the distances $z_{m^{\prime}}-z_{m}$ between planes. Note from the definition, Eq. (4), that this retardation is in fact accounted for in the lattice sum not through the explicit Bloch phase term $e^{i \mathbf{k}_{\|} \cdot \mathbf{R}_{m n}}$ but through the fact that one coherently sums spherical waves $\mathbf{G}^{0}$ at a given overall $k$. In the actual implementation of the lattice sum (see the Appendix), the retardation transverse to each plane appears explicitly [cf. Eq. (A3), in particular], as the different real and evanescent diffracted orders $\mathbf{k}^{g}$ that couple lattices each involve different phase slips $k_{z}^{\mathbf{g}}\left|z_{m^{\prime}}-z_{m}\right|$ with transverse wave vector $k_{z}^{\mathbf{g}}=\sqrt{k^{2}-\left|\mathbf{k}_{\|}+\mathbf{g}\right|^{2}}$.

Returning to the overall solution strategy, we note that the summation in the new, third term in Eq. (11) is no more difficult to deal with than the original lattice sum in Eq. (3) and Eq. (4). In terms of its overall structure, Eq. (11) is almost identical to the usual multiple scattering problem for just $N$ scatterers and is a simple set of $6 N$ linear equations for $6 N$ unknowns once one has calculated the required lattice summations that couple the lattices. However, while usually the coupling matrix would simply have $\boldsymbol{\alpha}_{m}^{-1}$ on the diagonal, now the block diagonal reads $\boldsymbol{\alpha}_{m}^{-1}-\mathcal{G}^{\neq}\left(\mathbf{k}_{\|}, 0\right)$. In other words, the polarizability of each scatterer is renormalized by the lattice sum of the layer in which it is embedded. The off-diagonal terms for a standard multiple scattering problem would simply be the Green function $\mathbf{G}\left(\mathbf{r}_{m}, \mathbf{r}_{m^{\prime}}\right)$ that quantifies the dipole field at $\mathbf{r}_{m}$ due to a dipole at $\mathbf{r}_{m^{\prime}}$. Here, the off-diagonal terms are given by the lattice-summed Green function $\mathcal{G}\left(\mathbf{k}_{\|}, \mathbf{r}_{0}^{m^{\prime}}-\mathbf{r}_{0}^{m}, z_{m^{\prime}}-z_{m}\right)$ that quantifies the field strength exerted by lattice $m^{\prime}$ on lattice $m$.

Once the dipole moments in each layer are retrieved, one can once again find the far-field intensity in each diffracted order, taking into account the fact that different layers are shifted to have the base point away from the origin, giving rise to a phase shift. The fields associated with each order are

$$
\left(\begin{array}{l}
\mathbf{E}^{\mathbf{g}} \\
\mathbf{H}^{\mathbf{g}}
\end{array}\right)=\frac{2 \pi k i}{A \cos \theta} \mathbf{M}(\theta, \phi) \sum_{m=1}^{N}\left(\begin{array}{c}
\mathbf{p}^{m} \\
\mathbf{m}^{m}
\end{array}\right) e^{-i \mathbf{k}^{\mathbf{g}} \cdot\left(\mathbf{r}_{0}^{m}, z^{m}\right)} .
$$

While exactly the same diffracted orders appear as for a single lattice, their amplitude is now a coherent summation of the diffracted fields from each layer separately. The coherence involves both the phase in $\left(\mathbf{p}_{m}, \mathbf{m}_{m}\right)$ that arises from the selfconsistent interactions and an additional phase slip owing to the displacement $\left(\mathbf{r}_{0}^{m}, z^{m}\right)$.

\section{DIFFRACTIVE CALCULATION}

Our calculation method can be applied to 2D lattices that have more than one scatterer per unit cell (complex basis for the lattice) $[1,10,11]$, as well as stacks of such $2 \mathrm{D}$ lattices that form a 3D structure. In this section we show calculations for 2D lattices with more than one scatterer per unit cell. As the first demonstration, we have calculated the grating diffraction efficiencies of comparatively dilute split ring lattices for two types of lattices. Lattice $\mathrm{A}$ is a simple square lattice (periodicity, $1500 \mathrm{~nm}$ ), while lattice B has every second split ring rotated by $180^{\circ}$ in checkerboard geometry [10]. These two 
lattices have an identical number of scatterers per unit area but have differently sized unit cells. In particular, the B lattice has a larger unit cell, thereby giving rise to extra allowed diffraction orders. These orders have nonzero amplitude only when the scatterers have an induced magnetic dipole and, hence, can be viewed as direct and background-free quantification of the magnetic SRR response. As split ring polarizability we include radiation damping according to

$$
\boldsymbol{\alpha}^{-1}=\boldsymbol{\alpha}_{\mathrm{LC}}^{-1}-i \frac{2}{3} k^{3} \mathbb{I}
$$

in the static LC-circuit polarizability

$$
\boldsymbol{\alpha}_{\mathrm{LC}}=\frac{\omega_{0}^{2} V}{\omega_{0}^{2}-\omega^{2}-i \omega \gamma}\left(\begin{array}{ccccc}
\eta_{E} & 0 & \ldots & 0 & i \eta_{C} \\
0 & & & & 0 \\
\vdots & & \ddots & & \vdots \\
0 & & & & 0 \\
-i \eta_{C} & 0 & \ldots & 0 & \eta_{H}
\end{array}\right)
$$

exactly as argued in Ref. [14]. This formulation ensures a scattering theory that satisfies reciprocity and the optical theorem in the limit of no Ohmic damping, while $\gamma$ introduces absorption loss. We assume the LC resonance to be described by a resonance frequency $\omega_{0}=1.26 \times 10^{15} \mathrm{~s}^{-1}$ (the corresponding free-space wavelength $\lambda=1500 \mathrm{~nm}$ ), a damping rate $\gamma$ equal to the Ohmic damping rate of gold $\left(\gamma=1.25 \times 10^{14} \mathrm{~s}^{-1}\right)$, and parameters $\left\{\eta_{E}, \eta_{H}, \eta_{C}\right\}=\{0.7,0.3,0.4\}$, where $V$ is the split ring volume $\left(150 \times 150 \times 30 \mathrm{~nm}^{3}\right)$. As the environment we assume a homogeneous medium of index $n=1.0$. Figure 2 reports the calculated zero-order transmission coefficient and the efficiencies for transmitted grating orders, assuming normal incidence and plotting only the intensity copolarized with the incident beam. A polarization analysis is presented below in the framework of our experiments. The zero-order transmission shows a broad minimum punctuated by a narrow grating anomaly around $6700 \mathrm{~cm}^{-1}$ (wavelength equal to the grating pitch). The transmission depth is around $20 \%$, commensurate with the very dilute nature of the grating and the per-split ring cross section [18] of around $0.3 \mu \mathrm{m}^{2}$. The grating anomaly is coincident with the emergence of the first-order grating diffraction at the grazing exit angle. The first-order grating diffractions reach efficiency values of around 0.5 to $1 \%$ at $\lambda^{-1}=7100 \mathrm{~cm}^{-1}$. For a simple lattice of electric dipoles polarized along the $x$ axis, one would expect the highest grating efficiency to occur for the $(0, \pm 1)$ order, owing to the fact that the single-dipole emission pattern is peaked away from the dipole orientation. For instance, for a frequency of $\lambda^{-1}=7100 \mathrm{~cm}^{-1}$, the grating orders appear circa $80^{\circ}$ from the plane normal. For this angle, the expected intensity ratio assuming purely electric dipole scatterers is $\sin ^{2} \theta: \cos ^{2} \theta=$ $8: 1$ for the $(0, \pm 1)$ orders compared to the $( \pm 1,0)$ orders. Reference [25] verifies that this reasoning for estimation of the grating diffraction efficiency from single-dipole radiation patterns indeed quantitatively holds for dilute lattices of plasmonic rod dipole antennas. The fact that the actual ratio calculated for the split ring lattice is closer to $2: 1$ than the estimated 8:1 is a direct consequence of the magnetic dipole radiation.

Next we turn to the superlattice. If the base lattice A has reciprocal lattice vectors $b_{1}=2 \pi / d(1,0)$ and $b_{2}=2 \pi / d(0,1)$, the superlattice can be classified as having a two-atomic basis with reciprocal lattice vectors $b_{1}=2 \pi / d(1 / 2,1 / 2)$ and $b_{2}=2 \pi / d(1 / 2,-1 / 2)$. Consequently, a set of extra diffraction orders appears. We label the extra orders with half-integer number pairs obtained by normalizing the $\mathbf{G}$ vector added to $\mathbf{k}_{\|}$in the diffraction process to $2 \pi / d$. It should be noted that the extra orders will not correspond to all combinations of half-integers. Indeed, the first set is at $( \pm 1 / 2, \pm 1 / 2)$ [and not at $( \pm 1 / 2,0)$ resp. $(0, \pm 1 / 2)]$. The calculated diffraction efficiencies contain two independent measures of the magnetic dipole component of SRR scattering. First, the amplitude in the
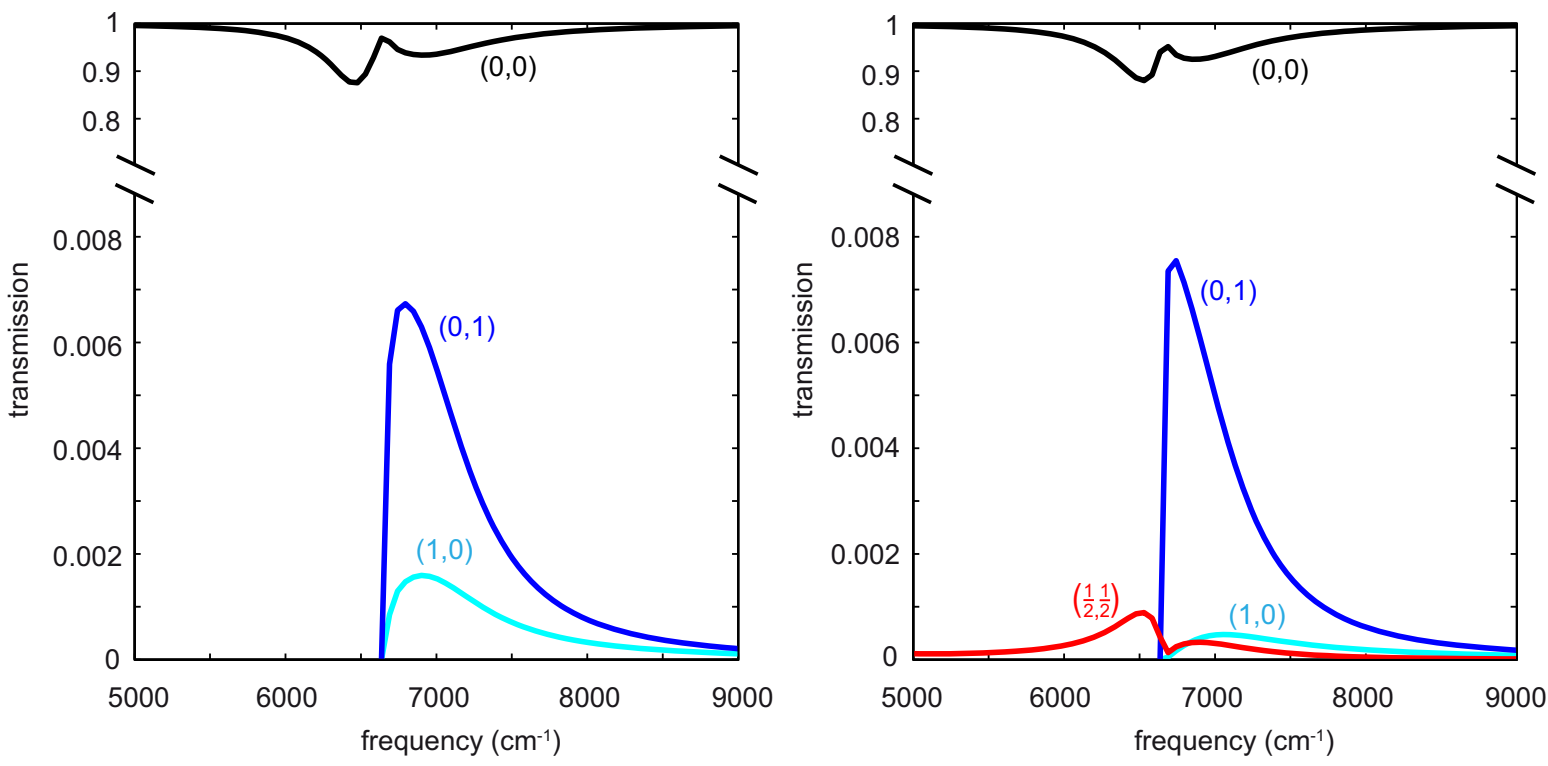

FIG. 2. (Color online) Calculated transmission for diffracted orders for SRR lattices of type A (left) and type B (right) assuming a lattice of 1500-nm pitch, in an environment of $n=1$. As SRR parameters we use $\omega_{0}=1.26 \times 10^{15} \mathrm{~s}^{-1}, \gamma=1.25 \times 10^{14} \mathrm{~s}^{-1}$, and $\left\{\eta_{E}, \eta_{H}, \eta_{C}\right\}=$ $\{0.7,0.3,0.4\}$. Note the emergence of half-integer diffraction orders for lattice type B, with their cutoff being outside of the presented frequency range. 
half-integer orders can be viewed as direct and backgroundfree quantification of the magnetic SRR response, since they would not appear for $\eta_{H}=\eta_{C}=0$. As Fig. 2 shows, the half-integer orders appear at frequencies $1 / \sqrt{2} \times 6700 \mathrm{~cm}^{-1}$ onwards and, indeed, contain significant amplitude (up to $2.5 \%$ total diffraction efficiency spread over four beams). The second measure of the magnetic dipole strength is evident in the $( \pm 1,0)$ and $(0, \pm 1)$ orders. As soon as the condition for emergence of the $( \pm 1,0)$ and $(0, \pm 1)$ is crossed, the $( \pm 1 / 2, \pm 1 / 2)$ orders diminish in amplitude. Remarkably, the contrast between the $(0, \pm 1)$ and the $( \pm 1,0)$ orders is much stronger than in the A lattice, reaching up to 10:1 rather than $2: 1$. This higher ratio is a direct consequence of the fact that, in the superlattice, the magnetic dipoles are aligned in antiphase in the two sublattices, while the electric dipoles are aligned in phase. Since the $( \pm 1,0)$ intensity for the A lattice is mainly from the $z$-oriented magnetic dipoles, the diminished $(1,0)$ diffraction strength is due to cancellation of the magnetic dipole contributions in the antiparallel arrangement [10].

\section{SETUP FOR EXPERIMENT}

In order to verify the predictions for diffraction by the A and B lattices, we perform a diffraction experiment in the near infrared on $\mathrm{Au}$ split ring resonator arrays fabricated using e-beam lithography and liftoff [26]. The dimensions are $150 \times 150 \times 30 \mathrm{~nm}$, with gaps of $50 \times 80 \mathrm{~nm}$. The setup (shown in Fig. 3) is essentially built as an infrared-range copy of the setup reported in Ref. [25] with achromatic lenses specified for 1050-1620 nm. Light from a supercontinuum source (Fianium) is frequency selected by passing through an acousto-optical tunable filter (Crystal Technology PCAOM NIR 2) that allows us to select any wavelength from 1100 to $2000 \mathrm{~nm}$, with a spectral selection FWHM bandwidth of around $12 \mathrm{~nm}$ at $1550 \mathrm{~nm}$. After passing a linear polarizer (Thorlabs LPNIR100) the beam is weakly focused to a spot of $\approx 20-\mu \mathrm{m}$ diameter, smaller than an individual lattice, which covers an area of $50 \times 50 \mu \mathrm{m}^{2}$. This illumination approximates a plane wave, as it has an angular spread $\Delta k_{\|} / k=0.02$. Light passing through the sample is collected by a $100 \times$ oil objective (Olympus UPLSAPO; NA $=1.4$ ). To retrieve $k$-space information, we image the back aperture of

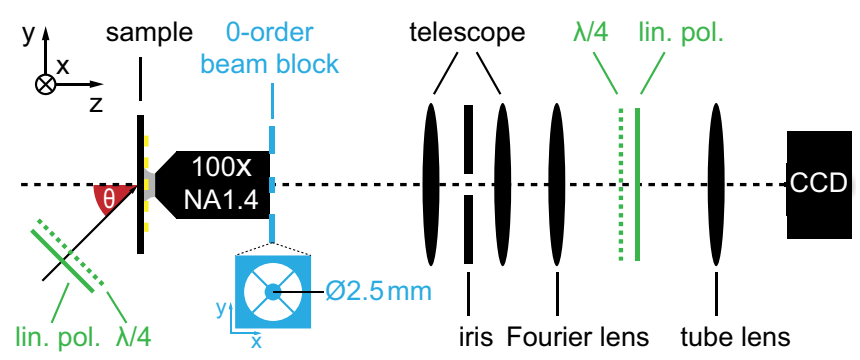

FIG. 3. (Color online) In our Fourier microscopy setup, light from a filtered supercontinuum light source is incident from the left at an angle $\theta$ from the lattice plane normal. A $100 \times$ immersion objective collects transmitted light. The iris provides real-space filtering prior to Fourier imaging. The beamstop is shown by the circle-within-square (blue) symbol. the objective onto an InGaAs CCD (Vosskühler NIR 300-PGE) via a $4 f$ imaging system $\left(f_{\text {telescope }}=50 \mathrm{~mm}, f_{\text {Fourier }}=f_{\text {tube }}=\right.$ $200 \mathrm{~mm})$. An iris placed in the real-space image plane in between the 1:1 telescope lens pair blocks light stemming from the edges of an illuminated lattice, which would cause additional features in the $k$-space image. Our aim is to study the intensity and polarization for nonzero diffracted orders. However, the sparse arrangement of scatterers will result in back focal plane images that are dominated by the zero-order transmitted beam. Indeed, the calculations in Fig. 2 indicate an intensity ratio of the order of $10^{3}: 1$, making acquisition of diffraction orders well above the noise, without oversaturation of the zeroth-order problematic on a CCD of limited dynamic range of 12 bits. We overcome this limitation by placing a zero-order beam block at the back aperture of the objective. The beam block is a metal sheet disk of 2.5-mm diameter supported by a cross of thin wires (200- $\mu \mathrm{m}$ width), made by electrical discharge machining in a $200-\mu \mathrm{m}$-thick metal foil. The size of the disk should be compared to the back aperture size of our Olympus UPLSAPO $100 \times$ objective, which is $7.1 \mathrm{~mm}$ in diameter. For cross-polarized excitation/analysis configurations the beam block may be omitted.

\section{DIFFRACTION MEASUREMENT}

First, we compare the diffraction patterns of the type A versus type B lattices (sketch in Fig. 4) under planewave illumination at $1550 \mathrm{~nm}$. We use lattices immersed in index matching oil to obtain a completely symmetric optical environment and use a pitch of $1250 \mathrm{~nm}$. The measurements are hence done well to the blue of the diffraction condition. Data in the copolarized channel, i.e., with input polarization and detection polarization along the split ring base $x$, clearly evidence the $( \pm 1,0)$ and $(0, \pm 1)$ diffraction orders. The efficiency in these four orders stands at an almost 1:1 ratio, in apparent contradiction to Fig. 2. However, it should be noted that at the wavelength used, the grating diffraction angle of about $55^{\circ}$ is much closer to the sample normal than in the calculation example. Consequently, the expected intensity ratio on the basis of electric dipoles alone is around $2: 1$, further reduced by magnetic contributions. The magnetic contribution to diffraction should appear much more clearly in cross-polarized detection, since the electric dipole should be $x$-polarized for all orders, while the magnetic dipole should give rise to $y$-polarized light for the orders on the $k_{x}$ axis (and vice versa). Indeed, in cross-polarized detection the $(0, \pm 1)$ orders almost vanish compared to the orders on the $x$ axis $( \pm 1,0)$ diffraction orders, yielding a 7:1 ratio.

For the B lattice, the diffraction pattern in copolarized excitation and detection is dominated by the $( \pm 1,0)$ and $(0, \pm 1)$ orders, as in the A lattice. This similarity, which is also apparent in Fig. 2, is indicative of the fact that actual split rings do not have equally electric, magnetic, and magnetoelectric polarizability but, in fact, are expected [19] to have $\alpha_{E}: \alpha_{H}: \alpha_{C}=$ $4: 1: 2$. Nonetheless, the extra half-integer orders are faintly visible in the copolarized Fourier image. The magnetic nature of the SRR scatterers is much more clearly evident in the crosspolarized Fourier image. In cross-polarization, all the integer orders almost vanish. For the $(0, \pm 1)$ orders such vanishing is expected since both the electric and the magnetic dipole 

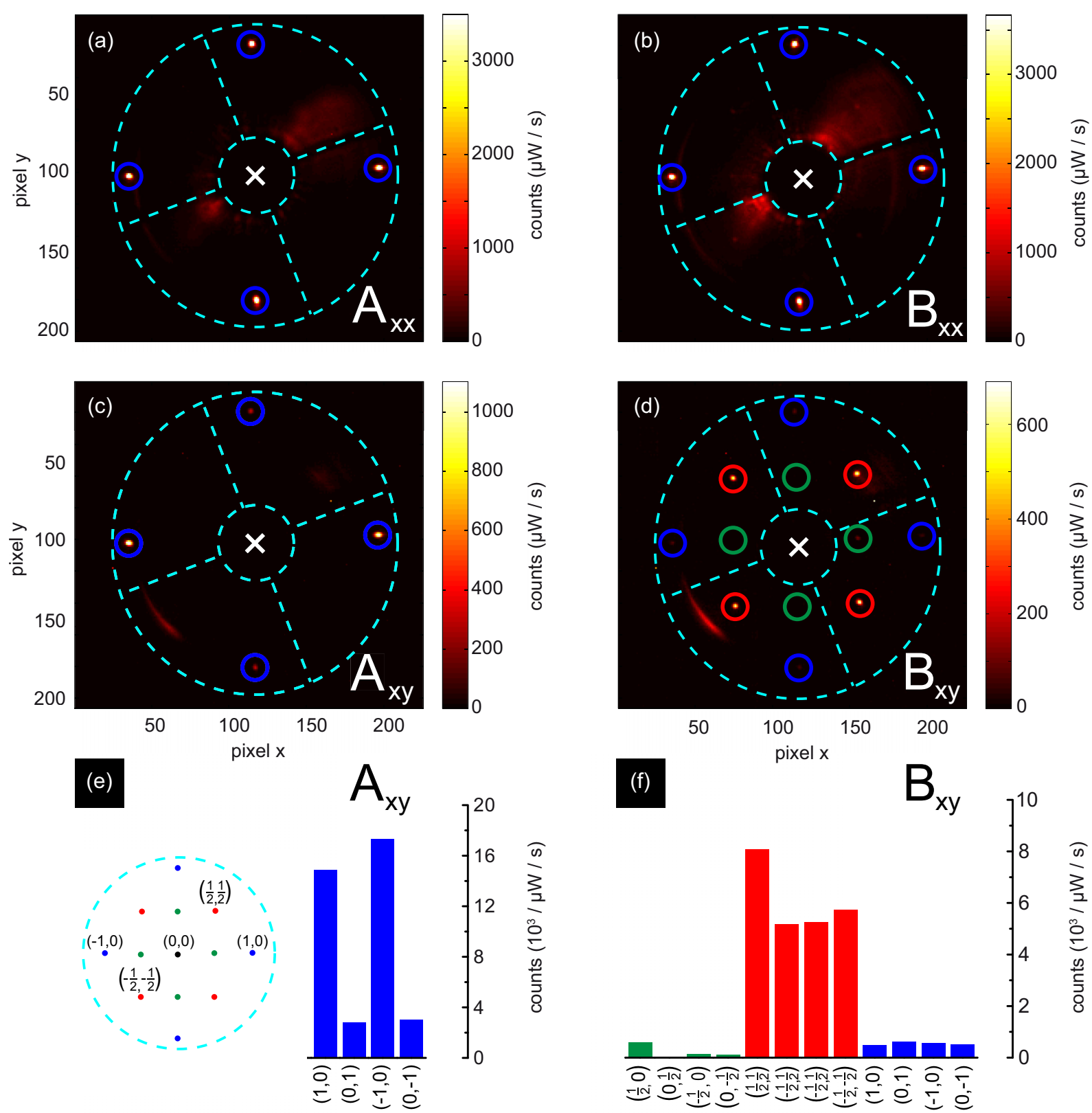

FIG. 4. (Color online) Diffraction pattern analysis for lattice type A (a, c) and lattice type B (b, d) for plane-wave illumination with $x$-polarized light at $1550 \mathrm{~nm}$. For the cross-polarized analyzer orientation ( $y$ direction) the diffraction patterns for lattice types A and B (c, d) are clearly distinct. For A, diffracted light is found in orders (blue) close to the maximum collection angle of the system (dashed circle). In contrast, lattice B also shows half-integer orders (red). Cross-polarized detection brings out these orders more clearly. Histograms (e, f) quantify the cross-polarized intensity. Low-diffraction-order intensities at positions indicated in green are attributed to minute deviations of SRR center positions from a perfect square lattice introduced during the manufacturing process.

contributions are $x$-polarized for wave vectors in the $(y, z)$ plane. For the $( \pm 1,0)$ orders, all $y$-polarized light must result from the magnetic dipole radiation. However, in strong contrast to the A lattice, where the magnetic dipoles are all aligned and give a clear $( \pm 1,0)$ diffraction, in the B-lattice cancellation occurs owing to the fact that the two distinct sublattices have magnetic moments in antiphase. Remarkably, in the crosspolarized data the half-integer diffraction orders clearly stand out at the $( \pm 1 / 2, \pm 1 / 2)$ nodes, providing a background-free measure for the presence of magnetic polarizability in the sin- gle SRR polarizability. Strictly, no intensity is expected at the $( \pm 1 / 2,0)$ and $(0, \pm 1 / 2)$ nodes if the split rings can be considered points located exactly on a square lattice. Careful inspection points to a very faint diffraction intensity at these angles, which we attribute to a minute deviation in split ring positioning introduced by the RAITH lithography pattern generator.

Next we turn to a more comprehensive polarization analysis of the half-integer orders. We study a lattice of higher split ring density (900-nm pitch), so that the diffraction orders move outwards in the Fourier image. In fact, at this pitch, the 

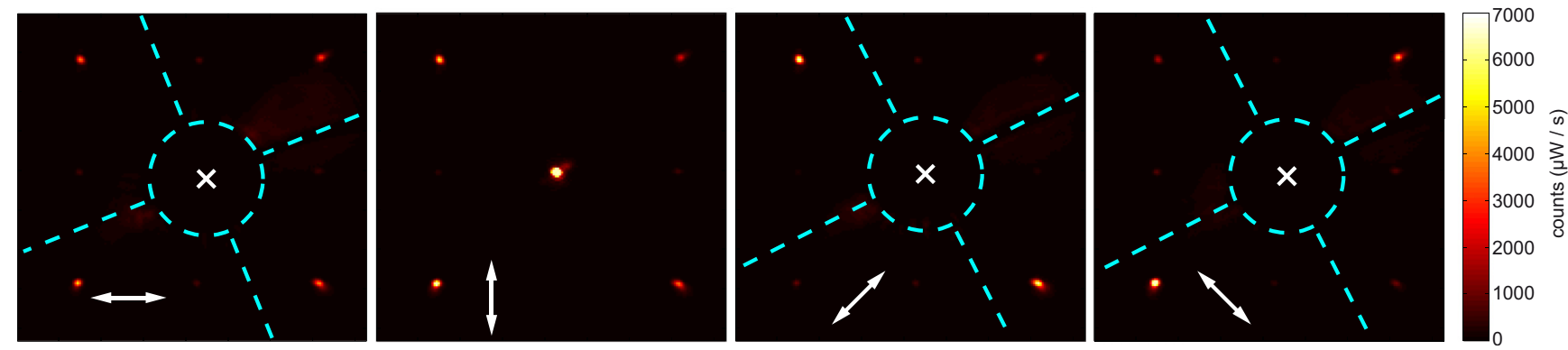

FIG. 5. (Color online) Experimental polarization analysis for lattice type B. For a lattice pitch of $900 \mathrm{~nm}$, only the half-integer orders are propagating. Fourier images for different analyzing polarizer settings (white arrow) reveal that the orders are tangentially polarized.

conditions for integer order diffraction $( \pm 1,0)$ are not yet met, while the half-integer orders occur at large NA, conveniently far from the zero-order transmitted beam. According to point scattering theory, if the split rings had no magnetic polarizability and no bianisotropy, they would all carry identical electric dipole moments and no magnetic moment, and the half-integer orders would have strictly zero intensity. From symmetry it is apparent that even in the presence of bianisotropy and split ring coupling, at normal incidence the induced electric dipole moments in each sublattice will still be equal. Consequently, the half-integer diffraction orders are solely due to the induced magnetic dipoles. Since in high-NA Fourier imaging of an out-of-plane magnetic dipole, the back focal plane image must contain a radially polarized magnetic field, polarization analysis of the Fourier image must reveal tangential polarization. Figure 5 shows Fourier images with excitation polarization along the split ring base $x$ and the detection polarizer set at $0,90^{\circ}$, and $\pm 45^{\circ}$ relative to the $x$ axis. The four orders indeed are dominantly tangentially polarized, commensurate with radiation of out-of-plane magnetic dipole moments.

\section{ANGLE-RESOLVED TRANSMISSION AND PSEUDOCHIRALITY}

Recent reports indicate that dense split ring lattices that have square symmetry and all split rings aligned show a strongly handed response when illuminated under an angle. This handed response was first noted for split ring lattices by Plum et al. [27-29], who proved that an asymmetric response is allowed under oblique incidence even for a geometrically nonchiral structure. The key to their argument is that the illumination geometry should be included in the symmetry considerations. A different interpretation is that the handed response is intrinsic to the single-split-ring polarizability tensor and directly results from the off-diagonal bianisotropy. This form of "pseudochirality" was discussed in 1997 by Tretyakov [30] in the framework of current carrying cut wires, $\Omega$ particles, and helices. Recently, this pseudochirality was measured to be exceptionally strong in split rings by Sersic et al. [19]. A shortcoming of that work was that, while the asymmetric response was attributed to the single building block, in fact the observation was made on lattices without mapping the influence of the lattice symmetry. Here, we present calculations as well as experiments on superlattices of different symmetry, i.e., the A lattice, with all split rings aligned, and the B lattice, in which bianisotropy should cancel in the zero-order transmission.

Figure 6 (left) shows calculations for the A lattice, assuming split rings in a square lattice of pitch $300 \mathrm{~nm}$, in surroundings index-matched to glass $(n=1.5)$. As parameters we take $\left\{\eta_{E}, \eta_{H}, \eta_{C}\right\}=\{0.7,0.3,0.4\}, \gamma=1.25 \times 10^{14} \mathrm{~s}^{-1}$, and $\omega=1.108 \times 10^{15} \mathrm{~s}^{-1}$ in order to match the resonance wavelength to the wavelength with the lowest normal incidence transmission dip. As also reported by Sersic et al. [19] and Lunnemann et al. [23] lattice A shows a strongly asymmetric handed response when rotating the sample in the incident beam around the split ring symmetry axis and collecting the total transmitted signal without analyzer. The asymmetry consists of a sharp decrease in transmission when going from normal incidence to positive angles at handed incidence light and an almost-vanishing of the transmission resonance when either rotating the sample in the opposite direction or collecting in the opposite helicity channel. For rotation around the $x$ axis, the asymmetry vanishes entirely. Here we have measured and calculated transmission for both the helicity conserving $\left[T_{ \pm \pm}(\theta)\right]$ channels and the helicity nonconserving channels $\left[T_{ \pm \mp}(\theta)\right]$. Calculations of transmission resolved for the output polarization shows that the handed transmission contrast is not associated with polarization conversion. Indeed, our calculation predicts a large contrast between $T_{++}$and $T_{--}$but no amplitude in the helicity nonconserving channel $\left(T_{+-}=0\right.$ and $\left.T_{-+}=0\right)$.

Our transmission measurement is effectuated in the same setup used for diffraction experiments. The detection path again uses an $N=1.4$ oil immersion objective and InGaAs CCD detector. Oblique illumination is provided by fiber coupling the spectrally filtered supercontinuum light and mounting the output fiber coupler, collimation and focusing optics, and polarization optics on a manually adjustable goniometer arm. We can select the transmitted beam from just the patterned sample area using real-space and Fourier-space filtering in the detection path. A reference for the transmission measurement is obtained by shifting the sample to image an unpatterned region.

Measurements of transmission for two handednesses for an index-matched lattice with 300-nm pitch of split rings $(150 \times 150 \times 30 \mathrm{~nm}$, with gaps of $50 \times 80 \mathrm{~nm})$ indeed show strong asymmetry in oblique incidence transmission for the A lattice. Compared to the previous report by Sersic et al. [19], the measured transmission asymmetry is clearly evident, yet less pronounced, which we attribute 

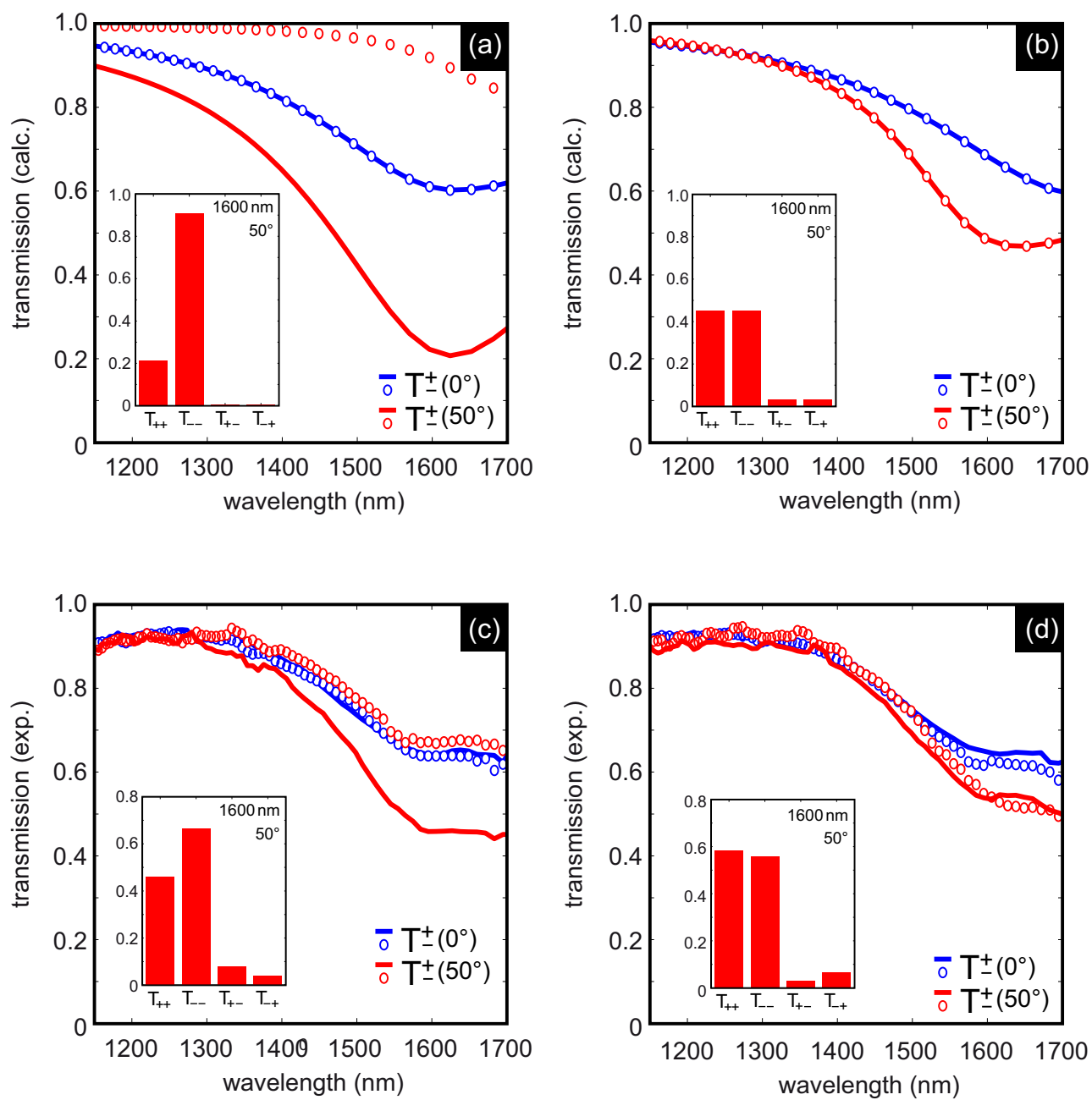

FIG. 6. (Color online) Calculated and experimentally retrieved transmission spectra for SRR lattices of type A (a, c) and type B (b, d) with lattice pitch $300 \mathrm{~nm}$. Right-handed (+) and left-handed (-) circularly polarized light is incident at $0^{\circ}$ (blue) and $50^{\circ}$ (red) from the lattice normal. Insets: Histograms for transmission at $1600 \mathrm{~nm}$ and a $50^{\circ}$ angle of incidence. The contrast in copolarized transmission channels $T_{++}$ and $T_{--}$for lattice type A (insets in a and c) vanishes for lattice type B (insets in b and d). Transmission for cross-polarized polarization channels $T_{+-}$and $T_{-+}$is considerably smaller for both lattice types compared to the copolarized case.

to the fact that in that measurement the substrate was not index matched. The presence of a dielectric substrate can significantly affect the magnetic polarizability and increase the bianisotropy as reported for split rings on substrates [31] as well as fishnet metamaterials [32]. Polarization analysis of the transmitted light at resonance $\lambda_{0}=1600 \mathrm{~nm}$ quantifies that the transmitted light does not show strong polarization conversion.

Figure 6 (top and bottom right) show transmission calculations and measured data for the B lattice. The handeddependent difference in angle-dependent transmission vanishes entirely, showing that in this particular type of structure it is the single building block that determines the asymmetric response, as opposed to spatial dispersion, which was recently proposed to mimic chirality [33]. A viable solution to reduce the apparent bianisotropy in metamaterials is hence to create superlattices with rotated copies of the same bianisotropic building block. However, the price is that the increased unit cell size causes diffractive effects already at lower frequencies.

\section{STACKED LATTICES}

Finally, we discuss the application of our multi-stack-point scattering theory to a problem of great interest in metamaterials research, i.e., the emergence of properties such as transmission, $\epsilon$ and $\mu$, as a function of the number of lattices that one stacks to obtain a truly 3D structure. The second context in which stacking is relevant is in the formation of stereometamaterials, in which identical lattices are stacked with split rings rotated from layer to layer, and in which retardation between layers may also occur [15]. We refer to Ref. [34] for a treatment of this model in electrostatic circuit theory amended to include a retardation phase. We suppose one could stack layers of split rings resonant at $1.5 \mu \mathrm{m}$ that are in 2D square lattices of $300-\mathrm{nm}$ pitch, with a spacing between layers of $300 \mathrm{~nm}$, so that a finite crystal of simple cubic symmetry is formed. As parameters we use $\eta_{E}=\eta_{H}=\eta_{C}=1, \gamma=8.3 \times 10^{13} \mathrm{~s}^{-1}$, and $\omega=1.256 \times 10^{15} \mathrm{~s}^{-1}$, which emulates the case of maximally magneto-electric cross-coupled SRRs, with a damping rate comparable to that of silver. Stacked fabrication of split ring lattices was reported by Liu et al. [15]. Figure 7 (left) 

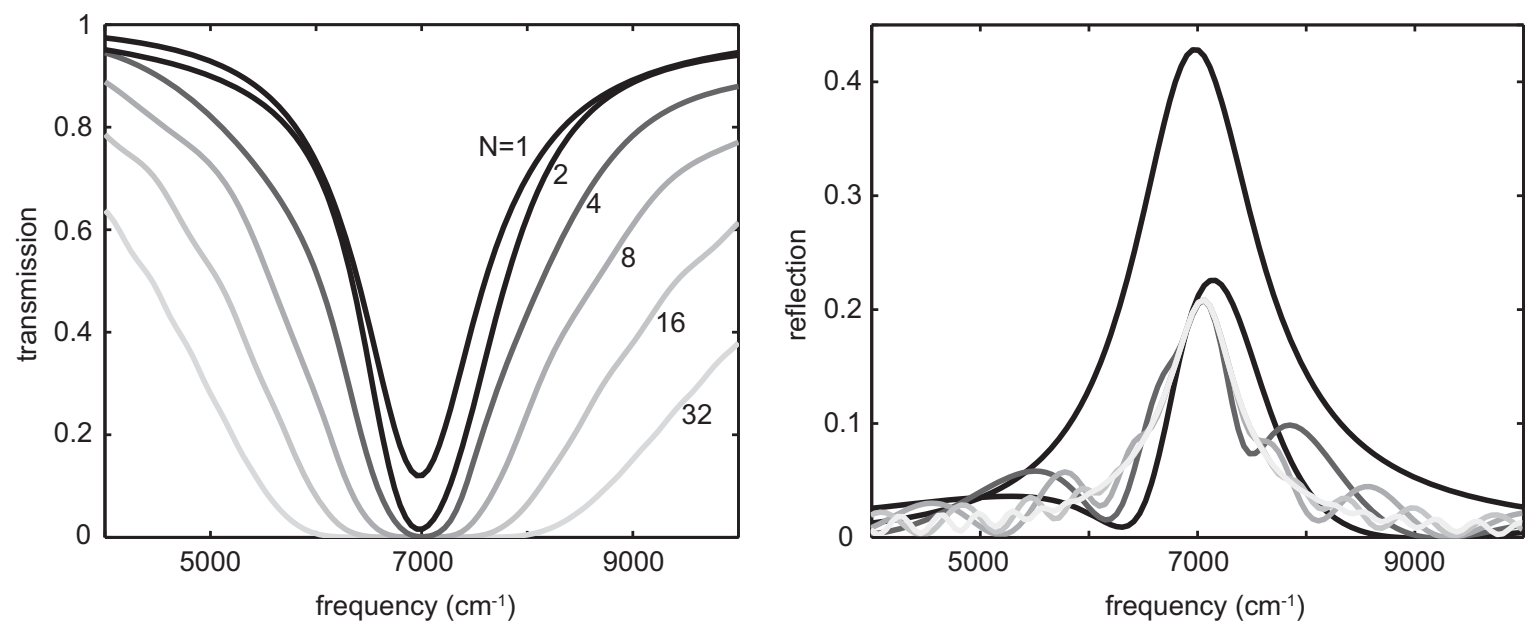

FIG. 7. Calculated transmission and reflection for $N$ stacked layers of lattice type A so that the $\operatorname{SRRs}\left(\eta_{E}=\eta_{H}=\eta_{C}=1\right.$, $\gamma=8.3 \times 10^{13} \mathrm{~s}^{-1}, \omega=1.256 \times 10^{15} \mathrm{~s}^{-1}$ ) are in a simple cubic arrangement with a lattice constant of $300 \mathrm{~nm}$.

shows the calculated transmission coefficient as a function of the number of layers. As one expects from reported data, a single layer already presents a significant suppression of transmission, to below $15 \%$, owing to the large cross section per split ring. The reflection coefficient of around $45 \%$ (Fig. 7) shows a peak complementary to the transmission, leaving a significant residual $40 \%$ absorption. As the number of layers is increased, the transmission stop gap significantly deepens and widens, as would be expected for an increasingly thick slab with a strong absorption resonance. At the same time, the reflection resonance decreases in strength, stays comparable in width to the single-layer reflection peak, and gains a significant number of fringes with increasing structure thickness. Since the multi-stack-point scattering theory returns full amplitude and phase information for reflection and transmission, one could continue to pursue a number of interesting studies on the convergence of $\epsilon$ and $\mu$ as retrieved from the reflection and transmission, as a function of the thickness, density, and single-dipole polarizability. Moreover, one could explore how multiple scattering interactions become apparent as spatial dispersion in the retrieved material constants.

\section{CONCLUSION}

In this work, we have successfully described experimentally retrieved grating order intensities and transmission spectra for diffractive and nondiffractive SRR lattices with a magnetoelectric point dipole model extended for stacks of $2 \mathrm{D}$ lattices applying Ewald's summation technique. The role of the magnetic dipole response for SRR lattices was revealed by comparing the diffraction order intensities of a lattice formed by a trivial unit cell (single-atomic base) to a lattice formed by a complex unit cell (two-atomic base) with alternating SRR orientation. The alternating SRR orientation in the complex unit cell case led to the absence of transmission contrast for right- and left-handed incident light for off-normal illumination as predicted by our model. Finally, we explored the influence of the finite number of $2 \mathrm{D}$ metamaterial layers forming a 3D stack on the stack's total transmission and reflection.

\section{ACKNOWLEDGMENTS}

This work is part of the research programme of the Foundation for Fundamental Research on Matter (FOM), which is part of The Netherlands Organisation for Scientific Research (NWO). A.F.K. acknowledges a NWO-Vidi fellowship.

\section{APPENDIX: LATTICE SUM}

The $6 \times 6$ dyadic Green function of free space can be obtained by applying the operator

$$
\left(\begin{array}{cc}
\mathbb{I} k^{2}+\nabla \otimes \nabla & -i k \nabla \times \\
i k \nabla \times & \mathbb{I} k^{2}+\nabla \otimes \nabla
\end{array}\right)
$$

to the Green function of the scalar-wave equation. Hence also the $6 \times 6$ dyadic lattice sum follows from the lattice sum for the scalar Green function, i.e., from

$$
\sum_{m, n} \frac{e^{i k\left|\mathbf{R}_{m n}-\mathbf{r}\right|}}{\left|\mathbf{R}_{m n}-\mathbf{r}\right|} e^{i \mathbf{k}_{||} \cdot \mathbf{R}_{m n}}=\Gamma^{(1)}+\Gamma^{(2)} .
$$

Note that in this defintion, $\mathbf{R}_{m n}$ are real-space lattice vectors strictly in the $x, y$ plane, while the observation point $\mathbf{r}=$ $(x, y, z)=\left(\mathbf{r}_{\|}, z\right)$ may be outside the lattice plane, i.e., away from $z=0$. On the right-hand side we have split the scalar lattice sum into a real-space and a reciprocal-space part that read as reported by Linton [24] as follows:

$$
\begin{aligned}
\Gamma^{(1)}= & \frac{\pi}{A} \sum_{\mathbf{g}}\left\{\frac { e ^ { i ( \mathbf { k } _ { \| } + \mathbf { g } ) \cdot \mathbf { r } _ { \| } } } { k _ { z } ^ { \mathbf { g } } } \cdot \left[e^{i k_{z}^{\mathbf{g}}|z|} \operatorname{erfc}\left(\frac{k_{z}^{\mathbf{g}}}{2 \eta}+|z| \eta\right)\right.\right. \\
+ & \left.\left.e^{-i k_{z}^{\mathbf{g}}|z|} \operatorname{erfc}\left(\frac{k_{z}^{\mathbf{g}}}{2 \eta}-|z| \eta\right)\right]\right\}, \\
\Gamma^{(2)}= & \sum_{\mathbf{R}}\left\{\frac { e ^ { i \mathbf { k } _ { \| } \cdot \mathbf { R } } } { 2 \rho _ { m n } } \cdot \left[e^{i k \rho_{m n}} \operatorname{erfc}\left(\rho_{m n} \eta+\frac{i k}{2 \eta}\right)\right.\right. \\
& \left.\left.+e^{-i k \rho_{m n}} \operatorname{erfc}\left(\rho_{m n} \eta-\frac{i k}{2 \eta}\right)\right]\right\} .
\end{aligned}
$$


The first term sums over all reciprocal lattice vectors $\mathbf{g}$ and, in terms of its dependence on the "observation" coordinate $\mathbf{r}$ at which it is evaluated explicitly, splits into in-plane and transverse coordinates $\left(\mathbf{r}_{\|}, z\right)$. All retardation effects in the plane appear explicitly through $\mathbf{k}_{\|} \cdot \mathbf{r}_{\|}$, while retardation transverse to the plane appears explicitly through $k_{z}^{\mathbf{g}}=\sqrt{k^{2}-\left|\mathbf{k}_{||}+\mathbf{g}\right|^{2}}$. The second part $\Gamma^{(2)}$ sums over real-space lattice vectors $\mathbf{R}$. In terms of the observation coordinate $\mathbf{r}$ at which it is evaluated, each summand is essentially in a spherical coordinate, with dependence only on the radius $\rho_{m n}=\left|\mathbf{R}_{m n}-\mathbf{r}\right|$. Note that since $\mathbf{r}=\left(\mathbf{r}_{\|}, z\right)$ has not only a component parallel to the plane but also one transverse to it, the $k \rho_{m n}$ terms account for retardation both in and transverse to the plane. As regards numerical implementation, the formulation holds for any choice of $\eta$; however, optimal convergence requires $\eta$ around $\sqrt{\pi} / a$, where $a$ is the lattice constant. Pointers as to how to apply the operator, Eq. (A1), and how many terms to sum are given in Ref. [23].
[1] A. V. Kildishev, A. Boltasseva, and V. M. Shalaev, Science 339, 1232009 (2013)

[2] X. Ni, S. Ishii, A. V. Kildishev, and V. M. Shalaev, Light Sci. Appl. 2, e72 (2013).

[3] N. Yu, F. Aieta, P. Genevet, M. A. Kats, Z. Gaburro, and F. Capasso, Nano Lett. 12, 6328 (2012).

[4] A. Alù and N. Engheta, J. Opt. A 10, 093002 (2008).

[5] F. Aieta, P. Genevet, M. Kats, N. Yu, R. Blanchard, Z. Gaburro, and F. Capasso, Nano Lett. 12, 4932 (2012).

[6] J. Sun, E. Timurdogan, A. Yaacobi, E. S. Hosseini, and M. R. Watts, Nature 493, 195 (2013).

[7] M. A. Kats, P. Genevet, G. Aoust, N. Yu, R. Blanchard, F. Aieta, Z. Gaburro, and F. Capasso, Proc. Natl. Acad. Sci. USA 109, 12364 (2012).

[8] C. M. Soukoulis, S. Linden, and M. Wegener, Science 315, 47 (2007).

[9] K. Busch, G. von Freymann, S. Linden, S. Mingaleev, L. Tkeshelashvili, and M. Wegener, Phys. Rep. 444, 101 (2007).

[10] N. Feth, M. König, M. Husnik, K. Stannigel, J. Niegemann, K. Busch, M. Wegener, and S. Linden, Opt. Express 18, 215 (2010).

[11] M. Decker, R. Zhao, C. M. Soukoulis, S. Linden, and M. Wegener, Opt. Lett. 35, 1593 (2010).

[12] C. Enkrich, M. Wegener, S. Linden, S. Burger, L. Zschiedrich, F. Schmidt, J. F. Zhou, T. Koschny, and C. M. Soukoulis, Phys. Rev. Lett. 95, 203901 (2005).

[13] C. Rockstuhl, T. Zentgraf, H. Guo, N. Liu, C. Etrich, I. Loa, K. Syassen, J. Kuhl, F. Lederer, and H. Giessen, Appl. Phys. B 84, 219 (2006).

[14] I. Sersic, C. Tuambilangana, T. Kampfrath, and A. F. Koenderink, Phys. Rev. B 83, 245102 (2011).

[15] N. Liu, H. Liu, S. Zhu, and H. Giessen, Nat. Photon. 3, 157 (2009).

[16] A. Serdyukov, I. Semchenko, S. Tretyakov, and A. Sihvola, Electromagnetics of Bi-anisotropic Materials: Theory and Applications, 1st ed., Electrocomponent Science Monographs, edited by D. de Cogan (Gordon and Breach Science, Amsterdam, 2001).
[17] F. J. García de Abajo, Rev. Mod. Phys. 79, 1267 (2007).

[18] M. Husnik, M. W. Klein, N. Feth, M. Koenig, J. Niegemann, K. Busch, S. Linden, and M. Wegener, Nat. Photon. 2, 614 (2008).

[19] I. Sersic, M. A. van de Haar, F. B. Arango, and A. F. Koenderink, Phys. Rev. Lett. 108, 223903 (2012).

[20] F. Bernal Arango and A. F. Koenderink, New J. Phys. 15, 1073023 (2013).

[21] M. Decker, S. Linden, and M. Wegener, Opt. Lett. 34, 1579 (2009).

[22] P. de Vries, D. V. van Coevorden, and A. Lagendijk, Rev. Mod. Phys. 70, 447 (1998).

[23] P. Lunnemann, I. Sersic, and A. F. Koenderink, Phys. Rev. B 88, 245109 (2013).

[24] C. Linton and I. Thompson, J. Comp. Phys. 228, 1815 (2009).

[25] I. Sersic, C. Tuambilangana, and A. F. Koenderink, New J. Phys. 13, 083019 (2011).

[26] I. Sersic, M. Frimmer, and A. F. Koenderink, Phys. Rev. Lett. 103, 213902 (2009)

[27] E. Plum, J. Zhou, J. Dong, V. A. Fedotov, T. Koschny, C. M. Soukoulis, and N. I. Zheludev, Phys. Rev. B 79, 035407 (2009).

[28] E. Plum, X. X. Liu, V. A. Fedotov, Y. Chen, D. P. Tsai, and N. I. Zheludev, Phys. Rev. Lett. 102, 113902 (2009).

[29] E. Plum, V. A. Fedotov, and N. I. Zheludev, J. Opt. 13, 024006 (2011).

[30] S. Tretyakov, C. Simovski, and A. Sochava, Advances in Complex Electromagnetic Materials, NATO ASI Series High Technology Vol. 28, edited by A. Priou, A. Sihvola, S. Tretyakov, and A. Vinogradov (Kluwer Academic, Dordrecht, The Netherlands, 1997).

[31] A. Kwadrin and A. F. Koenderink, Phys. Rev. B 87, 125123 (2013).

[32] Z. Ku, K. M. Dani, P. C. Upadhya, and S. R. J. Brueck, J. Opt. Soc. Am. B 26, B34 (2009).

[33] B. Gompf, J. Braun, T. Weiss, H. Giessen, M. Dressel, and U. Hübner, Phys. Rev. Lett. 106, 185501 (2011).

[34] H. Liu, J. X. Cao, S. N. Zhu, N. Liu, R. Ameling, and H. Giessen, Phys. Rev. B 81, 241403 (2010). 